\title{
La rééducation des filles en internat (1945-1965)
}

The Rehabilitation of Girls in Residential Institutions (1945-1965)

Die Umerziehung von Mädchen in Internaten (1945-1965)

La reeducación de las chicas en los internados (1945-1965)

\section{Anne Thomazeau}

\section{OpenEdition}

Journals

Édition électronique

URL : https://journals.openedition.org/histoire-education/1427

DOI : 10.4000/histoire-education. 1427

ISSN : 2102-5452

Éditeur

ENS Éditions

Édition imprimée

Date de publication : 1 septembre 2007

Pagination : 225-246

ISBN : 978-2-7342-1101-3

ISSN : 0221-6280

Référence électronique

Anne Thomazeau, «La rééducation des filles en internat (1945-1965) », Histoire de l'éducation [En ligne], 115-116 | 2007, mis en ligne le 01 janvier 2012, consulté le 20 mai 2021. URL : http://

journals.openedition.org/histoire-education/1427 ; DOI : https://doi.org/10.4000/histoire-education 1427

(c) Tous droits réservés 


\section{LA RÉÉDUCATION DES FILLES EN INTERNAT (1945-1965)}

par Anne THOMAZEAU

Les années d'après-guerre constituent une période déterminante pour le renouvellement des méthodes de prise en charge des mineurs délinquants ou inadaptés des deux sexes placés dans les institutions spécialisées. La volonté est alors forte de rompre avec le modèle hérité du XIX ${ }^{e}$ siècle et très vivement dénoncé lors des campagnes de presse contre les «bagnes pour enfants » de l'entre-deux-guerres (1). Les accusations concernent surtout des institutions pour garçons, mais celles qui accueillent les filles subissent également des critiques. D'une part, la plupart des établissements privés - largement majoritaires et le plus souvent gérés par des congrégations religieuses (2) semblent alors caractérisés par leur « immobilité » (3). En avril 1946, le directeur de l'Éducation surveillée signale que «beaucoup d'institutions sont restées telles qu'elles étaient il y a cinquante ans. Ce sont plutôt des garderies que des établissements de rééducation » (4). Cet avis est partagé par une religieuse du Bon Pasteur d'Angers, qui constate a posteriori qu' " en 1940, on en était resté, en partie, au

(1) Voir, notamment, Jacques Bourquin, « Une maison de correction. La colonie de Belle-Île-en-Mer, 1880-1945 », Revue d'histoire de l'enfance "irrégulière », hors série, juin 2007, p. 264.

(2) On dénombre entre 80 et 100 internats de rééducation pour filles entre 1945 et 1965 , dont seulement 1 à 3 sont publics. Les trois quarts sont gérés par des congrégations religieuses, en particulier celle du Bon Pasteur d'Angers, dont dépend environ la moitié des établissements; les établissements restants sont gérés par des associations laïques.

(3) Pascale Quincy-Lefebvre, Familles, institutions et déviances, Histoire de l'enfance difficile (1880-fin des années 1930), Paris, Economica, 1997, p. 224.

(4) Centre des archives contemporaines (désormais CAC) 910333-3, Plan de réforme des services de l'Éducation surveillée et des Institutions protectrices de l'Enfance en danger moral, présenté au Garde des Sceaux par le directeur de l'Éducation surveillée, avril 1946, p. 107. 
XIX ${ }^{e}$ siècle » (1). D'autre part, la seule institution publique féminine qui a survécu à la guerre, Cadillac, parait, en 1946, « impropre à sa destination d'établissement d'éducation » (2).

La volonté de réforme émerge donc dès l'entre-deux-guerres, s'épanouit sous l'Occupation, avant de devenir plus vive encore aux lendemains de la Seconde Guerre mondiale (3). En affirmant le primat de l'éducation sur la répression, l'ordonnance du 2 février 1945 sur l'enfance délinquante manifeste cette volonté de «moindre stigmatisation des mineurs délinquants » (4). Ces évolutions ne sont pas sans répercussions sur les établissements publics et privés de filles. La direction de l'Éducation surveillée tente vainement de réformer Cadillac avant de le fermer en 1952, à la suite, notamment, du suicide de Marguerite B., 19 ans (5). Entre-temps, un nouvel établissement public a été ouvert à Brécourt, dans le Val-d'Oise. À l'instar des établissements publics de garçons, Brécourt est présenté par la direction de l'Éducation surveillée comme un modèle, qui doit « serv[ir] d'exemple aux institutions privées » (6). Cependant, la volonté de réforme émane aussi du secteur privé. Ainsi l'élection, en 1940, d'une nouvelle supérieure générale à la tête du Bon Pasteur d'Angers semble avoir marqué un «tournant» (7) dans les orientations éducatives de cette congrégation. Aussi les congrégations religieuses réorganisent-elles peu à peu leurs établissements et modernisent-elles leurs méthodes, tandis que de plus en plus de religieuses obtiennent leur diplôme d'éducatrice. À mesure que le mouvement de réforme s'étend à l'ensemble du secteur, à un rythme variable selon les établissements, une nouvelle terminologie mettant l'accent sur la dimension éducative s'impose progressivement pour désigner ces

(1) Entretien avec une religieuse entrée au Bon Pasteur d'Angers dans les années 1930, 2004.

(2) Plan de réforme..., op. cit., p. 93.

(3) Michel Chauvière, Enfance inadaptée: l'héritage de Vichy, Paris, Éditions

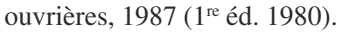

(4) Ludivine Bantigny, Le plus bel âge? Jeunes et jeunesse en France de l'aube des «Trente Glorieuses » à la guerre d'Algérie, Paris, Fayard, 2007, p. 147.

(5) Béatrice Koeppel, Marguerite B., une jeune fille en maison de correction, Paris, Hachette, 1987.

(6) Plan de réforme..., op. cit., p. 67.

(7) Sœur Giraud, "Le monde féminin du Bon Pasteur », in Mathias Gardet, Vincent Peyre, Françoise Tétard (dir.), Elles ont épousé l'éducation spécialisée. Éducatrices et femmes d'éducateurs il y a cinquante ans, Paris, L'Harmattan, 1999, p. 119. 
institutions et les distinguer des anciennes maisons de correction, de redressement ou de relèvement: ils sont appelés "internats de rééducation $»$.

Même si des traces de l'héritage carcéral restent visibles, comme la persistance de l'usage du « mitard » sous le nom de « cellule d'isolement », l'objectif de la rééducation en internat est bien, dans l'esprit de l'ordonnance de 1945, d'éduquer et non pas seulement de sanctionner. Cette orientation conduit les acteurs du secteur à préciser les principes éducatifs de la rééducation et donc à en définir les buts, les modèles et les méthodes. Ainsi, dès 1938, le réformateur de l'établissement public de garçons de Saint-Maurice précise-t-il :

«On ne peut dire que les principes de l'éducation des pervertis, des délinquants, soient différents de ceux de l'éducation des garçons normaux. Ils sont souvent les mêmes, et ne varient que dans leur application » (1).

Se trouve ainsi clairement explicitée la volonté, concernant également les filles, de replacer la prise en charge des mineur(e)s « dans une certaine normalité » (2), afin que les établissements de rééducation soient plus proches « du collège que de la prison » (3). Faut-il pour autant en déduire que les principes pédagogiques qui sous-tendent la rééducation des filles ne sont guère originaux au regard des modèles d'éducation féminine alors en vigueur? Ou bien les particularités réelles ou supposées des mineures placées impliquent-elles, au contraire, non seulement des variations dans l'application des principes, mais aussi le recours à des méthodes et à des images de référence singulières?

Afin de cerner les traits ordinaires et originaux de la rééducation des filles en internat, il convient de préciser, tout d'abord, pour quelles raisons les mineures ont été placées, raisons qui relèvent de la transgression de la loi mais aussi, et surtout, du non-respect des normes sociales, particulièrement celles de genre. Nous verrons ensuite que ces critères moraux et sexués impriment fortement leur marque sur la prise en charge de ces jeunes filles, comme le révèle

(1) Rapport de Vincent Hourcq à la commission technique, cité par Philippe ReyHerme, Quelques aspects du progrès pédagogique dans la rééducation de la jeunesse délinquante, Paris, Vrin, 1945, p. 136.

(2) Jacques Bourquin, « Une maison de correction. La colonie de Belle-Île-enMer... », art. cit., p. 265.

(3) Philippe Rey-Herme, op. cit., pp. 136-137. 
l'analyse, d'une part, des modèles féminins qui sous-tendent la rééducation - celui de la travailleuse et surtout de la maîtresse de maison et, d'autre part, celle des méthodes élaborées spécifiquement pour elles, telles que la fiction familiale et l'éducation morale, associées au silence sur le corps et la sexualité.

\section{DE L'INCONDUITE AU PLACEMENT}

Même si « la délinquance juvénile est considérée comme un phénomène intéressant avant tout les garçons » (1), la population des mineures placées en internat de rééducation n'est pas négligeable. Certes, entre la Libération et les années 1960, les filles ne représentent que 7 à $20 \%$ des délinquants juvéniles présentés aux juges des enfants, mais elles constituent un peu plus de la moitié de la population dite en danger (2). Or, il est pertinent de prendre en compte l'ensemble des activités des juges puisque les mêmes mesures éducatives, et notamment le placement, peuvent être appliquées aux deux catégories. Comme le nombre des procédures de protection, filles et garçons confondus, reste longtemps inférieur à celui des jugements pour délinquance, les filles représentent toujours moins du quart de la population jugée entre 1951 et 1959. L'équilibre se modifie après l'ordonnance de 1958 sur l'assistance éducative: ce type de procédure prend alors de l'ampleur, jusqu'à dépasser le nombre de jugements pour délinquance en 1961. Cette année-là et les suivantes, environ le tiers des jugements concerne des filles. Ce sont donc environ 4000 filles qui sont présentées aux juges, chaque année, avant l'application de l'ordonnance de 1958 sur l'assistance éducative, mais près de 20000 par an par la suite. De plus, les filles sont plus fréquemment placées que les garçons (3). En 1955, par exemple, 23,5\% des délinquantes faisant l'objet d'une mesure éducative et $57 \%$ des mineures jugées en danger sont placées en internat, contre, respectivement, $12,2 \%$ et 50,9\% pour les garçons. Aussi le nombre de lits dans les établissements de rééducation pour filles oscille-t-il

(1) Rapport annuel de la direction de l'Éducation surveillée, 1962, p. 195.

(2) Les éléments statistiques sont calculés à partir des données des Rapports annuels de la direction de l'Éducation surveillée.

(3) Parmi les mesures à la disposition du juge des enfants figurent l'admonestation, la remise aux parents sous le régime de la liberté surveillée, le placement (en centre d'observation, en foyer, en internat de rééducation), et enfin des sanctions pénales (incarcération, amende). 
entre 7000 et 8500 dans les années 1950 et 1960 (1). Cette sousreprésentation de la délinquance parmi les raisons du placement des filles par rapport à celles du placement des garçons se retrouve au sein des établissements. Les délinquantes représentent toujours moins de la moitié des mineures placées et cette proportion diminue au cours de la période, passant de près de $40 \%$ en 1952 à $13,5 \%$ en 1961. La majorité des jeunes filles placées le sont donc au titre d'une mesure de protection: vagabondage, correction paternelle et, à partir de l'ordonnance de 1958, assistance éducative (2). Ces intitulés recouvrent, en pratique, des situations très diverses: certaines jeunes filles accusées de vol ou même, pour l'une d'elles, de "vandalisme en bande » (3) sont, en fait, placées au titre des mesures de protection.

Pour tenter de cerner avec précision les raisons du placement d'une mineure, il est donc nécessaire de dépasser l'analyse en termes de catégories judiciaires et de chercher, au sein de son dossier individuel, le détail des faits qui ont conduit au placement, tels qu'ils sont rapportés par les différents intervenants (4). Il apparaît alors que la mesure de placement résulte souvent d'une volonté de contrôler le comportement sexuel de la mineure. D'une part, à l'origine des placements au titre des mesures de protection se trouvent fréquemment la crainte ou le soupçon d'un comportement sexuel déviant - avoir des rapports sexuels précoces, avec des partenaires multiples, voire se prostituer - ou même seulement potentiellement déviant - avoir de

(1) L'internat de Brécourt comporte environ 60 places. Dans les établissements tenus par des congrégations, la moyenne du nombre de lits diminue progressivement (140 lits en 1957, 130 lits en 1962).

(2) L'ordonnance du $1^{\text {er }}$ septembre 1945 sur la correction paternelle, développant les réformes adoptées par le décret du 30 octobre 1935, réglemente le recours à ce système: la compétence du juge des enfants est instituée et le placement par voie d'autorité est supprimé. Cette catégorie judiciaire disparaît avec l'ordonnance de 1958.

(3) AD Val-d'Oise, 688W 271, IPES de Brécourt, années 1960, dossier H 27, résumé du cas.

(4) Les dossiers ont un contenu très riche concernant aussi bien le passé des mineures (enquête sociale, actes judiciaires, rapports sur les placements antérieurs...) que le placement lui-même (notes d'observation, correspondance...). Pour la période 1945-1965, 216 dossiers individuels ont été étudiés, concernant 182 mineures. Plus précisément, trois années ont été sélectionnées (fin des années 1940, débuts des années 1950 et 1960) et le corpus est constitué de toutes les mineures entrées à Brécourt au cours de ces trois années (soit 70 individus) et de $10 \%$ des mineures entrées au centre d'observation de Chevilly-Larue au cours de ces trois mêmes années (soit 112 individus). Leur parcours a été complété, le cas échéant, par l'étude de leur dossier dans d'autres centres (établissements du Bon Pasteur et centre de Fresnes). 
mauvaises fréquentations, sortir le soir, fuguer. D'autre part, le comportement sexuel des jeunes filles placées au titre de l'ordonnance de 1945, donc suite à un acte de délinquance, est également examiné d'une façon quasi-systématique. Cette volonté de cerner le comportement sexuel est particulièrement visible dans les rapports d'observation des mineures placées au centre de Chevilly-Larue, qui comportent, systématiquement, un développement sur le comportement sexuel et un autre concernant le «sens moral» (1). À l'inverse, comme le souligne Ludivine Bantigny, « on ne trouve que très peu d'allusions, dans les jugements rendus sur les garçons, à leur moralité en matière sexuelle », comme si «le dévergondage [était] un attribut proprement féminin » (2).

La focalisation des acteurs sur cette question est telle que, malgré la diversité des situations, toutes les jeunes filles placées, sans exception, semblent être soupçonnées d'inconduite. Ainsi, en 1950, Odette Philippon qualifie indistinctement toutes les mineures placées de « débauchées » (3), tandis que, selon la directrice de l'Institution publique d'Éducation surveillée de Brécourt, « sauf en de rares exceptions, le malheur de nos pupilles a son origine dans les relations sexuelles » (4). Comme la délinquance des femmes adultes, étudiée par Claudie Lesselier pour la période antérieure à 1945, la délinquance, ou plutôt la déviance féminine juvénile est donc caractérisée par une « double transgression » (5), transgression légale mais surtout transgression morale. Par conséquent, restaurer la moralité des mineures fait partie des objectifs de la rééducation des filles. Celle-ci doit « prouver aux filles qu'il n'est pas nécessaire qu'elles aient des relations sexuelles avant leur mariage ou en dehors du mariage [et] les détourner de la prostitution » (6). Aussi les filles sont-elles plus souvent placées que les garçons et l'ouverture des établissements féminins sur l'exté-

(1) Cet établissement, géré par des religieuses de l'ordre de Notre-Dame de Charité, est le principal centre d'observation pour filles de la région parisienne.

(2) Ludivine Bantigny, op. cit., p. 142.

(3) Odette Philippon, La jeunesse coupable vous accuse. Les causes familiales et sociales de la délinquance juvénile, Paris, Sirey, 1950, p. 170.

(4) Archives du centre d'exposition «Enfants en justice» à Savigny-sur-Orge, sans cote, «L'éducatrice d'IPES », compte rendu d'une conférence de la directrice de l'IPES de Brécourt, session de formation des éducateurs, 1947.

(5) Claudie Lesselier, « Les femmes et la prison, 1820-1939 », in Jacques-Guy Petit (dir.), La Prison, le bagne et l'histoire, Paris, Les Méridiens, 1984, p. 116.

(6) «L'éducatrice d'IPES », op. cit. 
rieur est-elle moins rapide, afin de les maintenir à distance des éventuelles tentations et des mauvaises fréquentations. Toutefois, cette définition morale de la déviance juvénile féminine n'est pas le seul motif du placement en internat. Il est également justifié par les carences de leur famille et de leur milieu: la direction de l'Éducation surveillée qualifie les mineures placées de « jeune[s] fille[s] qui, par définition, n' [ont] pas bénéficié d'un milieu éducatif » (1). Le placement en internat de rééducation doit permettre de compenser et de corriger une éducation familiale déficiente, en s'appuyant plus particulièrement sur la formation professionnelle et sur l'enseignement familial et ménager.

\section{UN AVENIR DE TRAVAILLEUSE OU DE FEMME AU FOYER?}

La rééducation en internat poursuit deux objectifs principaux : former des travailleuses et des maîtresses de maison.

\section{Apprendre un métier}

Le trait le plus saillant de la rééducation est la formation professionnelle, pour les filles comme pour les garçons. Les différents acteurs ne cessent d'insister sur son importance, présentant la formation professionnelle comme l' « élément de base d'une bonne rééducation » (2). L'objectif n'est donc plus de faire travailler les mineur(e)s mais de leur dispenser un enseignement réellement qualifiant, validé, dans la mesure du possible, par l'obtention d'un diplôme. Cette nouvelle orientation émerge dans les années 1930, mais concerne d'abord surtout les garçons: Pascale Quincy-Lefevre souligne que « dans les années 1930, une congrégation comme celle du Bon Pasteur [...] se définit comme une "œuvre de Réforme et non d'enseignement professionnel" » (3).

(1) Rapport annuel de la direction de l'Éducation surveillée, 1962, p. 211.

(2) Conservatoire national des archives et de l'histoire de l'éducation spécialisée (désormais CNAHES), fonds Riehl (fonds en cours de classement et consulté sous dérogation, comme la plupart des archives citées), " Formation professionnelle des filles », IPES de Brécourt, $c .1956$, p. 8.

(3) Pascale Quincy-Lefebvre, op. cit., p. 219. 
Cette restriction n'a plus cours après guerre et, même si le travail à proprement parler ne disparaît pas totalement, les différentes congrégations placent progressivement la formation professionnelle au cœur de leur prise en charge. L'évolution est particulièrement rapide dans les Institutions publiques d'Éducation surveillée (IPES), de filles ou de garçons, qui, selon la direction de l'Éducation surveillée, " deviennent de plus en plus des centres d'apprentissage, équipés suivant les besoins d'une école professionnelle » (1). La formation professionnelle est, effectivement, au cœur de la prise en charge à l'IPES de Brécourt dès son ouverture, en 1947, et les termes de «pupilles » et de "pénitentes » sont assez rapidement remplacés par le mot d'élèves dans l'ensemble du secteur. Toutefois, dans le cas des filles, une argumentation paraît nécessaire pour justifier cette orientation, alors que la vocation domestique des femmes est, souvent, considérée comme naturelle:

«La place de la femme est avant tout au foyer. Pourtant, beaucoup de femmes sont obligées de travailler, soit que leur travail soit indispensable pour compléter les ressources du ménage, soit qu'elles vivent seules et qu'elles soient, parfois, des mères célibataires. La formation professionnelle des filles placées en internat doit pour cela tenir une place primordiale dans la rééducation $[\ldots] »(2)$.

Bien plus, dans le cas des filles, l'apprentissage d'un métier semble considéré comme une protection contre le risque de prostitution à la sortie: selon Odette Philippon, « le métier sera leur gagnepain et la sauvegarde de leur honneur » (3).

Puisque l'objectif est de gagner sa vie après la sortie, l'essentiel n'est pas de compléter la formation générale, mais bien d'apprendre un métier. Certes, certaines mineures reçoivent un enseignement scolaire pour préparer le certificat d'études primaires, mais les établissements n'offrent que rarement la possibilité de poursuivre des études générales secondaires. Les instructions provisoires sur l'enseignement général dans les IPES sont, d'ailleurs, explicites: " l'enseignement général reste subordonné à l'enseignement professionnel » (4).

(1) Rapport annuel de la direction de l'Éducation surveillée, 1951, p. 27.

(2) Rapport annuel de la direction de l'Éducation surveillée, 1962, p. 196.

(3) Odette Philippon, op. cit., p. 172.

(4) CAC 91 0300-51, Instructions provisoires sur l'Enseignement général dans les Institutions publiques, Direction de l'Éducation surveillée, 14 novembre 1946. 
Cette priorité donnée à l'apprentissage d'un métier est, bien sûr, en lien aussi avec le parcours scolaire antérieur des élèves et leur âge, majoritairement supérieur à 16 ans (1).

La formation professionnelle en internat de filles n'est pas seulement un principe pédagogique, elle est aussi une réalité, et une réalité de plus en plus large, puisqu'un nombre croissant d'établissements préparent leurs élèves à des diplômes officiels (2). La préférence est donnée aux certificats d'aptitude professionnelle (CAP), mais les jeunes filles qui ne paraissent pas aptes à l'obtenir préparent différents diplômes jugés plus accessibles, tels que le certificat d'aptitude aux métiers et, progressivement, le certificat de formation professionnelle des adultes. Comme dans l'ensemble de l'enseignement technique féminin, ces différents diplômes correspondent à trois principaux groupes de métiers: métiers du vêtement et du textile (lingerie, couture...), emplois de bureaux (secrétariat, sténodactylo...), et enfin emplois de maison (arts ménagers...) ou de service, comme la coiffure (3). L'exemple de l'emploi du temps suivi à Brécourt, sans modifications majeures entre 1947 et la fin des années 1960, permet de mieux cerner la place prépondérante de la formation professionnelle dans la rééducation en internat. Ainsi, en 1967, les jeunes filles commencent leur journée par deux heures de formation professionnelle théorique en classe; elles vont ensuite en atelier de 10 heures à 12 heures et de 14 heures à 17 heures 30 , avec une coupure récréative vers 16 heures. Enfin, de 17 heures 30 à 18 heures 30, elles suivent une heure d'enseignement général (4). Les journées des mineures placées sont donc essentiellement occupées par la formation professionnelle.

Évidemment, toutes les mineures placées en internat n'obtiennent pas pour autant un diplôme. Selon le rapport de la direction de l'Éducation surveillée de 1962, pour l'ensemble des établissements privés,

(1) Bien que les établissements étudiés puissent accueillir des mineures de 14 à 21 ans, celles de 16 à 18 ans sont sur-représentées, d'autant plus que la proportion d'élèves âgées de plus de 19 ans diminue, passant de 27,3\% des mineures placées dans les internats de rééducation privés en 1957 à 9,1 \% en 1964.

(2) Rapport annuel de la direction de l'Éducation surveillée, 1962, p. 222.

(3) Certains établissements maintiennent une formation agricole. Les formations suivies dans les internats de garçons correspondent, elles, à des métiers réputés masculins: mécanique, bâtiment...

(4) CAC 2000 0111-7, IPES de Brécourt, Emploi du temps, 1967. 
un quart environ des filles placées obtiennent une qualification professionnelle (1). Cependant, à en croire les témoignages d'anciennes éducatrices et instructrices techniques, beaucoup de mineures appréciaient particulièrement cette possibilité de suivre une formation professionnelle qualifiante et souhaitaient profiter de cette opportunité, ce qui est parfois perceptible à la lecture de leurs dossiers. Ainsi, dans une lettre à sa mère, Solange, après avoir raconté qu'elle a un « cafard énorme », précise à propos de sa formation en coiffure: « je crois que c'est la seule chose qui me retienne à Brécourt car si non ce ferais longtemps que je n'y serais plus » (2). Cette appréciation positive portée sur la formation professionnelle est évidemment en lien avec la situation antérieure des mineures : moins d'un quart avait suivi une formation professionnelle, tandis que la majorité avait déjà travaillé, généralement comme employées de maison, ouvrières ou vendeuses. Le placement offre donc à ces jeunes filles, souvent issues de milieux populaires, la possibilité de bénéficier d'une formation professionnelle de qualité. Solange, par exemple, qui souhaitait apprendre la coiffure après son certificat d'études primaires et n'avait pu le faire faute de moyens suffisants, obtient son CAP de coiffure après deux années de placement à Brécourt (3). Considérant des parcours similaires, une religieuse du Bon Pasteur va jusqu'à présenter la délinquance comme une « aubaine », en décrivant le cas d'une élève:

« Elle n'aurait jamais bénéficié d'une orientation professionnelle dans son milieu, tant à cause de sa situation de fille cadette ayant derrière elle toute une pléiade de frères et sœurs, qu'à l'incurie et à l'inconscience de ses parents. [...] La délinquance a été pour elle une aubaine qui lui a ouvert de nouveaux horizons » (4).

Cependant, l'objectif reste traditionnel. D'une part, « les métiers enseignés devront être les métiers de base permettant aux jeunes élèves des établissements de rééducation d'accéder aux emplois normalement ouverts aux jeunes filles de leur milieu et de leur niveau » (5). D'autre part et surtout, cette prépondérance de la forma-

(1) Rapport annuel de la direction de l'Éducation surveillée, 1962, p. 98.

(2) AD Val-d'Oise, 688W 271, IPES de Brécourt, années 1960, dossier H27, lettre retenue au dossier. Tous les prénoms ont été modifiés et les citations extraites des dossiers sont recopiées sans correction orthographique.

(3) AD Val-d'Oise, 688W 271, IPES de Brécourt, années 1960, dossier H27.

(4) Sœur Marie de Saint Robert, Place et limite de l'orientation professionnelle au centre d'observation, mémoire pour l'obtention du diplôme d'éducatrice, Bon Pasteur d'Angers, 1953, p. 64.

(5) Rapport annuel de la direction de l'Éducation surveillée, 1962, p. 210. 
tion professionnelle n'est pas la conséquence d'un rejet, par les acteurs de la rééducation, du modèle traditionnel de la femme au foyer, modèle particulièrement valorisé, en ce temps de baby-boom, par un discours dominant occultant la présence ancienne et forte malgré un certain infléchissement - des femmes sur le marché du travail (1). Au contraire, ce modèle apparaît comme la principale référence de la rééducation.

\section{2. « Le but lointain de presque toutes est le mariage » (2)}

Même si une formation professionnelle réellement qualifiante est dispensée, les acteurs de la rééducation des filles ne cessent d'affirmer que l'internat de rééducation a également pour mission de « préparer [les élèves] à leur futur rôle de maîtresse de maison, en veillant à la formation familiale et ménagère » (3). Ainsi sœur Marie de Saint Robert remarque-t-elle que les mineures placées « auront toutes à exercer la formation ménagère qu'elles auront reçue, bonne ou mauvaise. Tandis que les garçons sont marqués par leur profession, la femme devrait être marquée surtout par sa vocation féminine » (4). Dans le même esprit, la directrice de l'IPES de Brécourt explique qu'il est nécessaire, en plus de la formation professionnelle, de « prévoir une formation familiale, ménagère, sociale et morale, adaptée à la vocation féminine » (5). Bien plus, les acteurs semblent considérer que le mariage et la maternité légitime sont davantage les signes d'une rééducation réussie que les succès professionnels. Sœur Marie du Christ, religieuse du Bon Pasteur d'Angers, écrit, à propos de jeunes filles «s'étant orientées uniquement vers une activité professionnelle », qu' " il est nécessaire qu'elles s'orientent vers une vie normale, autrement elles perdront en l'espace de quelques mois tout

(1) Voir Sylvie Schweitzer, Les femmes ont toujours travaillé. Une histoire du travail des femmes aux XIXe et XX' siècles, Paris, Éditions Odile Jacob, 2002. Sur la prégnance du modèle de la mère au foyer après-guerre, voir notamment Yvonne Knibiehler, La révolution maternelle depuis 1945. Femmes, maternité, citoyenneté, Paris, Perrin, 1997, Partie I, et Christine Bard, Les femmes dans la société française au XXe siècle, Paris, Armand Colin, 2003, pp. 184-190.

(2) Rapport annuel de la direction de l'Éducation surveillée, 1962, p. 207.

(3) CAC 2000 0111-7, IPES de Brécourt, Emploi du temps, 1967.

(4) Sœur Marie de Saint Robert, op. cit., p. 64.

(5) CAC 91 0162-5, «L'institution professionnelle d'Éducation surveillée pour filles de 16 à 21 ans », note interne, Direction de l'Éducation surveillée, s.d. (après 1967). 
le bénéfice de leur séjour »(1). Malgré la volonté de réforme et la place de la formation professionnelle, la perception de l'avenir des jeunes filles placées semble donc largement s'inscrire dans une logique de prédestination domestique des femmes. Alors que, dans les années 1960, «le discours officiel, tant politique que médiatique, reste traditionnel, exaltant la vocation maternelle et la femme au foyer »(2), il paraît bien difficile pour les acteurs de se distancier d'un modèle d'éducation féminine hérité du XIX ${ }^{\mathrm{e}}$ siècle. Aussi les objectifs de l'internat de rééducation peuvent-ils être rapprochés de la volonté de "former l'épouse modèle » qui caractérise les maisons d'éducation de la Légion d'honneur au XIXe siècle (3).

Quant aux jeunes filles elles-mêmes, même si les faits qui leur sont souvent reprochés - inconduite, rapports sexuels - pourraient laisser penser qu'elles se sont affranchies de certaines normes sociales sexuées, leurs aspirations semblent très proches de celles des filles non placées. Comme la plupart des « jeunes filles du babyboom, [elles] hésitent [...] entre conformité aux normes, conjugaison des rôles et émancipation » (4). En effet, leurs réponses au sujet de rédaction "Comment envisagez-vous votre vie future? ", posé au centre d'observation de Chevilly-Larue, laissent apparaitre leurs hésitations, mais aussi la prégnance du modèle non pas tant de la femme que de la mère au foyer. Ainsi, pour Bernadette, le travail semble être une situation provisoire avant le mariage et surtout la maternité: « D'abord apprendre un métier. Ensuite me marier avoir des enfants et les élever » (5), tandis qu'Annick est plus hésitante: « Je me vois maîtresse d'un foyer tranquille auprès de mon mari [...]. S'ils [mes enfants] ont 5 ou 6 ans, je travaillerais peut-être, s'ils ont moins seul mon mari travaillera au dehors » (6).

(1) Sœur Marie du Christ, Formation professionnelle et rééducation, mémoire pour l'obtention du diplôme d'éducatrice, Bon Pasteur d'Angers, 1955, p. 47.

(2) Anne-Marie Sohn, Âge tendre et tête de bois. Histoire des jeunes des années 1960, Paris, Hachette, 2001, p. 219.

(3) Rebecca Rogers, Les demoiselles de la Légion d'honneur. Les maisons d'éducation de la Légion d'honneur au XIXe siècle, Paris, Éditions Plon, 1992, chapitre X: « Former l'épouse modèle ».

(4) Anne-Marie Sohn, op. cit., p. 219.

(5) Archives du centre de Chevilly-Larue, dossier 028-1038, années 1960, rédaction.

(6) Archives du centre de Chevilly-Larue, dossier 066-1229, années 1960, rédaction. 
Le projet d'avenir qui sous-tend la rééducation des filles en internat ne parait donc pas radicalement différent de celui qui est proposé aux filles non placées. D'ailleurs, l'ensemble de l'enseignement technique féminin est caractérisé, comme la formation professionnelle dans les internats de rééducation, par « une ambiguïté constante entre l'aspect professionnel de l'enseignement dispensé et son aspect domestique et moral »(1), comme l'indiquent la valeur et le sens donnés à l'enseignement ménager. Celui-ci peut être réellement professionnalisant: il permet notamment d'obtenir le CAP d'Arts ménagers, utile pour travailler dans l'hôtellerie, la restauration, dans les collectivités ou encore dans le secteur médico-social (2). Pour autant, son objectif affiché semble bien être de "produire des anges du foyer » (3). D'ailleurs, selon la loi du 18 mars 1942, validée à la Libération, «l'enseignement ménager familial » est obligatoire pour toutes les jeunes filles, quelle que soit leur orientation professionnelle, et tous les CAP féminins comprennent des épreuves d'éducation ménagère (4). Dans les internats de rééducation, l'importance donnée à la formation ménagère est encore plus grande. En effet, les mineures sont, en général, considérées comme ignorant jusqu'aux rudiments de l'art ménager, comme le constate sœur Marie du Christ:

« À l'arrivée, tout ou presque est à apprendre; il n'est pas rare de voir des jeunes filles, même de milieu très modeste, qui n'ont jamais épluché de légumes ou tenu une aiguille. M. [...] avoue [...] n'avoir jamais cassé un œuf, épluché salade ou carottes; elle a 18 ans » (5).

Toutes les mineures placées suivent donc des cours d'enseignement ménager. Bien plus, certaines préparent des concours destinés aux ménagères: la direction de l'Éducation surveillée signale, dans son rapport annuel de 1957, «que la "Fée du Logis" de 1955 était une élève d'un établissement habilité » (6).

(1) Patrice Pelpel, Vincent Troger, Histoire de l'enseignement technique, Paris, L'Harmattan, 2001, p. 194.

(2) Marianne Thivend, «Les formations techniques et professionnelles de filles. Le cas lyonnais aux XIX ${ }^{e}$ et $\mathrm{XX}^{\mathrm{e}}$ siècles », in Marianne Thivend (dir.), Apprentissage et formations techniques et professionnelles de filles et de garçons, XIX ${ }^{e}-X X^{e}$ siècles, Lyon, Cahiers Pierre Léon, n 6, 2005, pp. 31-32.

(3) Id.

(4) Gérard Bodé, L'enseignement technique de la Révolution à nos jours, T. II: 1926-1958, Vol. 1 : Textes officiels, Paris, INRP/Economica, 2002, pp. 69-70.

(5) Sœur Marie du Christ, op. cit., p. 14.

(6) Rapport annuel de la direction de l'Éducation surveillée, 1957, p. 128. 
Des rapprochements sont donc possibles entre les internats de rééducation et les établissements d'enseignement technique. Cependant, les limites d'une telle comparaison sont mises en évidence par la directrice de Brécourt elle-même:

« Ce serait un non-sens que de vouloir comparer une IPES à un internat de l'Éducation nationale [...], internat où les élèves sont heureux d'être acceptés..., d'où l'on part en week-ends et en vacances pendant plus d'un tiers de l'année..., un internat qui n'est qu'un à-côté de la vie familiale... » (1)

Alors que les instances d'éducation et de socialisation sont multiples pour les jeunes non placé(e) s - famille, école, mouvements de jeunesse, etc. -, la mission explicitement assignée à l'internat de rééducation est d'assurer, pendant la durée du placement, une prise en charge totale, afin d'être une sorte de « substitut parental» (2). Or, l'éducation familiale et ménagère est supposée être transmise surtout par et dans la famille: « au cours d'une étroite symbiose, la mère inspire des gestes et des attitudes, elle cultive plus ou moins consciemment des habitudes et des sentiments » (3). Dans le cas des mineures placées, cette mission revient donc à l'institution.

\section{PRÉPARER À « UNE EXISTENCE NORMALE DE MAÎTRESSE DE MAISON » (4)}

Préparer les filles placées à leur futur rôle d'épouse et de mère, c'est-à-dire réaliser leur éducation familiale à l'extérieur du cadre familial, implique l'élaboration et la mise en œuvre de méthodes éducatives spécifiques.

(1) CAC 91 0162-5, « L'institution professionnelle d'Éducation surveillée pour filles de 16 à 21 ans », note interne, Direction de l'Éducation surveillée, s.d. (après 1967).

(2) Jacques Bourquin, «Saint-Maurice, colonie pénitentiaire agricole. Le temps de la réforme: 1934-1936-1950 », Revue d'histoire de l'enfance "irrégulière », hors série, juin 2007, p. 277.

(3) Yvonne Knibiehler et al., De la pucelle à la minette, les jeunes filles de l'âge classique à nos jours, Paris, Temps actuels, 1983, pp.105-106.

(4) Rapport annuel de la direction de l'Éducation surveillée, 1962, p. 195. 


\section{La fiction familiale}

Pour réaliser l'éducation familiale des jeunes, deux principes sont particulièrement appliqués: la pédagogie de groupe et l'éducation par l'exemple. Selon le principe de la pédagogie de groupe, qui émerge dès les années 1930 mais s'épanouit surtout aux lendemains de la Seconde Guerre mondiale, les élèves, filles ou garçons, ne doivent pas être traités collectivement sous la responsabilité de l'ensemble des éducateurs, mais être subdivisés en petits groupes placés sous l'autorité d'un ou de quelques éducateurs. Ce principe, mis en place à l'IPES de Brécourt dès son ouverture, s'impose progressivement dans l'ensemble du secteur, même si la forte proportion d'établissements féminins hérités d'avant-guerre et initialement « aménagés pour une vie communautaire » (1) en ralentit l'application.

Cette pédagogie de groupe est supposée contribuer, pour les filles et les garçons, à « la préparation à la vie en société » (2):

«En internat, par la force des choses, le groupe doit durer; il se constitue, se soude, s'améliore peu à peu par l'effort de chacun. Cet effort, pour s'intégrer, être toléré, occuper une place, jouer un rôle, est éminemment formateur du caractère et de la volonté. Le groupe est donc un entraînement (au sens sportif du terme) à la vie dans la société des adultes [...]» (3).

Toutefois, dans le cas des filles, l'organisation en groupes a surtout pour objectif la mise en place d'un « système familial réel » (4). Cette référence à la famille n'est pas totalement absente de la rééducation masculine, mais elle concerne principalement les filles, comme le souligne la direction de l'Éducation surveillée en 1957: «Les établissements pour jeunes filles et pour adolescentes tentent de se rapprocher de ce modèle familial. Les établissements de garçons sont organisés de façon différente » (5). D'ailleurs, le nombre idéal de mineurs par groupes n'est pas le même selon le sexe: une vingtaine en moyenne pour les garçons, mais seulement dix à douze pour

(1) Ibid., p. 223.

(2) Ibid., p. 211.

(3) Ibid., p. 210.

(4) CNAHES, fonds Riehl, "Fonctionnement d'une institution professionnelle d'Éducation surveillée de filles, Brécourt », c. 1970.

(5) Rapport annuel de la direction de l'Éducation surveillée, 1957, p. 96. 
les filles (1). L'équipe de filles est ainsi à l'image d'une famille nombreuse, que l'on quitte pour suivre sa formation professionnelle et pendant les loisirs, et qui se recompose pour les repas et la nuit. Quant à l'éducatrice, elle partage le gîte et le couvert de «ses » filles, à la façon d'une maîtresse de maison et d'une mère de famille.

Afin de permettre « une formation empirique par la vie de tous les jours » (2), cette « organisation "familiale" » (3) doit être associée à un second principe éducatif: la pédagogie par l'exemple. Selon la directrice de Brécourt, «nos filles ne s'éduquent que par l'exemple, par identification aux modèles qui leur sont donnés » (4). De ce fait, « tout est vain si le personnel éducateur n'est pas hautement qualifié et sévèrement sélectionné sur le plan moral » (5). Ainsi, « seule une éducatrice bien au courant elle-même du travail de maîtresse de maison et capable de donner l'exemple au point de vue tenue et manières fera du bon travail » (6). En résumé, " l'éducatrice remplace la mère de famille, doit jouer ce rôle à la perfection afin de montrer et prouver l'avantage d'un foyer bien tenu, afin de créer des habitudes par la mise en pratique constante des théories » (7). Dans les institutions privées, la rééducation est également pensée sur un modèle maternel. Le rôle des éducatrices dans les institutions religieuses est ainsi évoqué par Odette Philippon: "chaque jour, la "mère" étudie les réactions, les anomalies, les défaillances, comme les progrès de son "enfant" ", et la directrice de l'établissement est qualifiée de "mère de toutes les "familles" » (8), c'est-à-dire de tous les groupes de jeunes.

Cette pédagogie par l'exemple concerne aussi la rééducation des garçons. Ainsi, Henri Joubrel, une des figures du secteur, précise que

(1) Rapport annuel de la direction de l'Éducation surveillée, 1957, p. 97, et Andrée Algan, Enquête sur la rééducation en institution des filles délinquantes et inadaptées qui font l'objet d'une décision judiciaire, Service de recherche de l'Éducation surveillée, Vaucresson, 1966, p. 23.

(2) Rapport annuel de la direction de l'Éducation surveillée, 1962, p. 210.

(3) $I d$.

(4) CNAHES, fonds Riehl, « La rééducation des filles, l'expérience de Brécourt », s.d.

(5) CAC 2000 0111-7, «Les établissements de rééducation de filles de Brécourt à Labbeville par Nesles-la-Vallée », document de présentation, c. 1962.

(6) «L'éducatrice d'IPES », op. cit.

(7) $I d$.

(8) Odette Philippon, op. cit., p. 171. 
l'éducateur doit être tel que "les enfants puissent le prendre en exemple en tout et pour tout, trouver en lui le "héros vivant" dont ils ont besoin » (1). Cependant, l'application de ce principe n'a dans les internats de filles et de garçons ni les mêmes modalités, ni les mêmes conséquences. D'abord, l'exemple à donner aux filles et aux garçons n'est évidemment pas le même. Tandis que l'éducatrice doit jouer le rôle de la mère de famille exemplaire, l'éducateur doit faire preuve de « bonté virile » et «maitriser sa conduite » (2). Ensuite, la plupart des éducatrices, même celles qui ne sont pas religieuses, sont célibataires puisqu'elles ne peuvent pas «être à la fois mère de famille dans [leur] propre foyer et mère de famille des huit adolescentes avec [...] lesquelles elle[s] doi[vent] former un véritable foyer familial modèle » (3). Et puisqu'elles sont célibataires, elles se doivent également d'être chastes, pour donner l'exemple de la jeune fille sérieuse. À l'inverse, la vie de groupe et l'application de la pédagogie par l'exemple impliquent, idéalement, le mariage pour les éducateurs. D'une part, la répartition des rôles sexués leur permet de constituer leur propre foyer et d'en confier la tenue à leur épouse à la manière des marins (4). D'autre part, un certain soupçon pèse, passé un certain âge, sur l'homme célibataire qui souhaite travailler auprès d'enfants et de jeunes gens (5). Le modèle à incarner par l'éducateur n'est donc pas celui de la chasteté, mais celui du foyer uni et fidèle.

L'organisation familiale dans laquelle les filles placées forment une famille autour d'une mère de substitution est donc au cœur de la rééducation féminine. Cependant, il s'agit bien d'une fiction, et d'une fiction très imparfaite. D'abord, les sanctions utilisées en internat ne peuvent être assimilées aux punitions familiales. Ainsi, l'envoi au «mitard», généralement appelé isolement, reste-il utilisé au moins jusqu'à la fin des années 1960, aussi bien dans les établissements privés que publics. De même, sont employées des punitions reposant sur l'humiliation, en particulier par la privation des attributs de la féminité, comme la coupe

(1) Henri Joubrel, «L'éducateur spécialisé », Revue de l'Éducation surveillée, $\mathrm{n}^{\circ} 5,1946$, p. 43.

(2) $I d$.

(3) «L'éducatrice d'IPES », op. cit.

(4) La comparaison est celle de l'épouse d'un éducateur: «Le point de vue de la femme de l'éducateur », Liaisons, ${ }^{\circ}$ 1, décembre 1951, reproduit dans Mathias Gardet, Vincent Peyre, Françoise Tétard (dir.), Elles ont épousé..., op. cit., p. 19.

(5) Henri Joubrel, art. cit., p. 42. 
des cheveux ou le port d'une «tenue de punie » (1). Voici comment Josette décrit à ses parents, avec révolte, les punitions reçues au retour d'une fugue, au début des années 1960:

« Jeudi en 15, jour de mon anniversaire, je suis allée au cachot jusqu'à dimanche soir, ensuite je suis rentrée dans mon équipe avec les vêtements de punies, c'est-à-dire grosses galoches en bois, jupe déchirée et dégoûtante et pull en laine râpeuse tout troué, avec ça, je n'avais plus qu'à m'appuyer contre le mur d'une église et tendre la main » (2).

L'ambiance en internat de rééducation n'est donc pas comparable en tous points à une atmosphère familiale. Quant aux réactions des mineures face à cette fiction, elles sont difficiles à apprécier mais semblent caractérisées par leur diversité. Ainsi, Simone envoie-t-elle régulièrement, après sa sortie, des cartes à l'occasion de la fête des mères, non à son éducatrice de groupe, mais à la directrice de Brécourt, " car pendant 4 ans vous avez été pour moi comme une mère » (3). À l'inverse, sœur Giraud explique que chez certaines mineures, « cela provoquait une révolte intérieure » (4).

\section{Quelle éducation sentimentale et sexuelle?}

Quelles qu'en soient les limites, cette fiction familiale peut, sans doute, effectivement, participer à la formation ménagère et domestique des mineures. En revanche, il convient de s'interroger sur son efficacité concernant l'éducation sentimentale et affective de ces jeunes filles. Pour devenir une épouse et une mère de famille accomplie, un préalable est évidemment nécessaire: le mariage et, auparavant, la rencontre du futur époux. Pour préparer cette rencontre, les mineures ne reçoivent pas, ou quasiment pas, d'éducation sexuelle avant les années 1970 (5). Cela n'est d'ailleurs pas propre aux inter-

(1) Christiane Pecriaux, Le Couvent à sabots, témoignage autobiographique, Nantes, Éditions Amalthée, 2005, p. 13.

(2) AD Val-d'Oise, 688W 489, IPES de Brécourt, années 1960, dossier T20, lettre retenue au dossier. Les galoches en bois devaient empêcher la mineure de courir, et donc de fuguer à nouveau.

(3) AD Val-d'Oise, 688W 270, Brécourt, années 1950, dossier H26, lettre de la mineure à la directrice.

(4) Soeur Giraud, op. cit., pp. 117-118.

(5) Béatrice Koeppel, De la pénitence à la sexologie. Essai sur le discours tenu aux jeunes filles, Paris, Le Sycomore, Vaucresson, Centre de formation et de recherche de l'Éducation surveillée, 1982. 
nats de rééducation, puisque, à la même époque, l'éducation sexuelle de la plupart des jeunes filles reste relativement limitée, malgré les efforts d'organismes comme l'École des Parents (1). L'Éducation nationale elle-même ne donne un cadre officiel à l'éducation sexuelle dans l'institution scolaire qu'en 1973, avec la « circulaire Fontanet». En revanche, ce qui paraît plus spécifique aux mineures placées, c'est la rareté de leurs occasions de contact avec des jeunes de l'autre sexe. En effet, surtout avant le milieu des années 1960, les internats de rééducation sont des institutions relativement fermées. Si les sorties collectives sont régulières, les occasions de sortir sans accompagnement sont rares et les jeunes filles placées n'ont que peu de contacts directs avec l'extérieur, et donc avec des hommes. Il est vrai que certaines bénéficient de permissions estivales dans leur famille ou bien participent à des camps de vacances, parfois mixtes, organisés par des associations extérieures. Ainsi, au début des années 1960, Yvonne, après deux années de séjour à Brécourt, part faire du ski avec l'Union nationale des centres de montagne (UNCM), ce qui est l'occasion de tester son comportement et donc d'évaluer l'avancée du processus de rééducation. À son retour, un compte rendu est envoyé au juge:

« Elle vient de participer à un stage de ski à Barèges, avec l'UNCM, stage mixte, dont elle est revenue en bonnes dispositions et pas du tout perturbée. Elle paraît avoir su rester sur le plan de la bonne camaraderie avec les garçons » (2).

Malgré quelques expériences de ce type, les possibilités de sortie des filles placées en internat de rééducation sont limitées par comparaison avec celles des autres jeunes filles, même celles qui sont internes dans un établissement scolaire et qui ont, elles, la possibilité de retourner à leur domicile pendant les week-ends ou, à défaut, pendant les vacances. La situation des mineures placées est donc différente de celle de la plupart des filles de leur âge, qui peuvent parfois échapper à la surveillance des adultes. D'autre part, les possibilités d'être en contact avec des hommes à l'intérieur de l'internat sont également faibles avant les années 1960: les religieuses auxquelles est confiée la majorité des mineures sont évidemment des femmes et le personnel laïc à Brécourt ou dans les établissements tenus par des

(1) Yvonne Knibiehler, "L'éducation sexuelle des filles au Xx siècle », Clio. Histoire, femmes et société, $\mathrm{n}^{\circ}$ 4, Le temps des jeunes filles, 1996, consulté en ligne.

(2) AD Val-d'Oise, 688W 273, Brécourt, années 1960, dossier H33, lettre au juge des enfants. 
congrégations est très majoritairement féminin. Quelques hommes travaillent à Brécourt (jardinier, personnel d'entretien...), mais ils n'occupent pas des fonctions éducatives. Les seuls hommes qui ont des réelles occasions de rencontre et de contact avec les mineures dans les différents établissements sont les prêtres ou aumôniers, qui interviennent régulièrement, et les médecins, en particulier les psychiatres. Les occasions de contact avec des hommes jeunes sont donc quasi-nulles en dehors des périodes de permission ou des fugues.

Cette volonté de marquer une coupure avec l'extérieur caractérise aussi la rééducation des garçons mais, dans le cas des filles, elle est accentuée par la façon dont est perçue leur déviance. Rappelons, en effet, qu'au moins une partie des mineures ont justement été placées parce qu'elles semblaient trop côtoyer les jeunes du sexe opposé et risquaient donc de devenir des jeunes filles « perdues ». La coupure avec le monde extérieur et la mise à distance des garçons, ainsi que le silence sur le corps et la sexualité, ne sont donc pas justifiés par la volonté de protéger l'ignorance et l'innocence des jeunes filles, comme cela pouvait être le cas pour les " oies blanches » au XIX siècle (1) ou même, pour une partie d'entre elles, durant les années 1940 à 1960. Il s'agit, au contraire, de leur faire « oublier le passé, les plaisirs vulgaires, la débauche » (2). Bien plus, l'intérêt porté par certaines de ces jeunes filles à la sexualité - la direction de l'Éducation surveillée parle même, pour les mineures prostituées, de «besoins [...] sur le plan sexuel » (3) - est réputé tel qu'il impliquerait le risque d'un développement de relations sexuelles entre filles au sein de l'internat. Il est, bien sûr, difficile de trouver des traces explicites concernant ces pratiques homosexuelles, d'autant plus qu'il est rarement possible, à la lecture des dossiers, de distinguer une amitié jugée suspecte d'une véritable relation amoureuse entre filles, avec ou sans rapports sexuels. Au total, seules 7 filles sur les 182 étudiées se voient explicitement reprocher leur homosexualité et 10 autres en sont soupçonnées. Certaines l'évoquent d'ailleurs explicitement, comme Ginette, qui raconte, au cours de son entretien d'accueil à Chevilly, sa relation homosexuelle lors de son précédent placement, à Brécourt:

(1) Yvonne Knibiehler et al., op. cit., chapitre 6: «Les oies blanches ».

(2) Dominique Riehl, "Psychologie de l'adolescente à sa sortie de l'Institution publique d'Éducation surveillée », Sauvegarde de l'enfance, n 3-4, 1950, p. 175.

(3) Rapport annuel de la direction de l'Éducation surveillée, 1962, p. 203. 
« J'ai des mœurs spéciales, comme on dit [...] Je ne peux pas oublier Huguette avec tout ce que nous avons fait ensemble pendant plus d'un an. [...] Je sais que ce que nous avons fait n'est pas normal mais c'est plus maintenant que je pourrai m'en séparer » (1).

Ce qui, en revanche, apparaît très clairement à la lecture des dossiers, c'est que les affinités et les rapprochements entre les élèves sont attentivement observés afin de repérer les amitiés dites douteuses et d'éviter leur évolution en relations homosexuelles, par exemple en changeant une des deux mineures concernées d'équipe. Ainsi, Sarah, dans une lettre à son assistante sociale sur Brécourt, écrit-elle: " lorsque l'on nous voit deux filles ensemble les dames avec leur esprit mal tournés pensent que l'on fait des bêtises je n'aime pas cela » (2). Le placement dans un environnement quasi-exclusivement féminin ne paraît donc pas considéré comme une condition suffisante au contrôle du comportement sexuel de ces mineures.

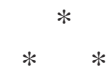

La singularité de l'éducation des filles en internat réside donc plus dans l'élaboration et l'application de méthodes spécifiques, adaptées aux caractéristiques attribuées aux mineures et aux conditions de leur placement, que dans la définition d'une identité féminine originale. En effet, les modèles d'éducation et la conception du rôle social féminin qui sous-tendent la rééducation semblent correspondre aux représentations alors en vigueur concernant les rôles sociaux de sexe, même si, dans le cas des filles placées, les aspects traditionnels sont particulièrement prégnants et persistants. Finalement, les contradictions perceptibles dans la rééducation féminine, telle que la complexe articulation entre la formation professionnelle et la promotion de la femme au foyer, sont sans doute le reflet, partiellement déformé, des tensions entre plusieurs conceptions du rôle des femmes au sein de la société d'après-guerre, conceptions oscillant entre assignation domestique et vocation maternelle d'un côté, épanouissement professionnel et émancipation de l'autre.

(1) Archives du centre de Chevilly-Larue, dossier 198-1886, années 1950, entretien d'accueil.

(2) AD Val-d'Oise, 688W 55, Brécourt, années 1950, dossier B61, lettre retenue au dossier. 
Réciproquement, l'évolution des identités sexuées et des modèles d'éducation féminine, qui s'accélère dans les années 1960, n'est pas sans répercussions sur le secteur de la rééducation des filles. Les principes pédagogiques en vigueur dans l'internat, relativement stables, malgré quelques inflexions, entre les années 1940 et le milieu des années 1960, sont ensuite progressivement remis en cause, une «nouvelle "génération" d'éducateurs » (1) - et d'éducatrices - les jugeant de plus en plus inadéquats. C'est alors l'ensemble du modèle de la prise en charge en internat, ainsi que la définition des méthodes et des objectifs de la rééducation, qui se trouvent critiqués. En particulier, des éducatrices contestent l'efficacité de la mise à l'écart des hommes et de la prégnance du modèle domestique. Déjà, au début des années 1960, une éducatrice en stage à Brécourt choisit comme thème, pour son mémoire de diplôme, la question suivante: "Comment peut-on remettre des filles sur pieds alors que c'est manifestement leurs rapports aux hommes qui, en gros, sont en question (pas que ça, mais quand même en gros), alors qu'il n'y a pas d'hommes? » (2). Les critiques s'accentuent ensuite en même temps que le regard porté sur la question de la sexualité des élèves se modifie: celle-ci cesse peu à peu d'être au cœur des préoccupations, et il apparaît de plus en plus nécessaire de compléter l'éducation morale par une réelle éducation sexuelle. Ces contestations multiples ont contribué à l'évolution de la prise en charge des mineures à partir de la fin des années 1960, et notamment à l'augmentation progressive des sorties libres, sans accompagnement, à l'introduction de la mixité dans l'équipe éducative et même parfois parmi les élèves, et au développement de l'éducation en milieu ouvert, c'est-à-dire dans le milieu de vie habituel des jeunes.

Anne THOMAZEAU

Doctorante ENS-LSH et Paris IV-Sorbonne

(1) Francine Muel-Dreyfus, Le métier d'éducateur, Paris, Éditions de Minuit, 1983, p. 184.

(2) Entretien avec une éducatrice-stagiaire à l'IPES de Brécourt au début des années 1960, 2006. 